\title{
2'-Deoxycytidine Decreases the Anti-tumor Effects of 5-Fluorouracil on Mouse Myeloma Cells
}

\author{
Ayano IwAZAKI* and Masanori YoshIOKA \\ Faculty of Pharmaceutical Sciences, Setsunan University; 45-1 Nagaotoge-cho, Hirakata, Osaka 573-0101, Japan.
} Received November 26, 2009; accepted March 7, 2010; published online March 11, 2010

2'-Deoxycytidine (dCyd), a pyrimidine nucleoside found at high concentrations in the plasma of cancer patients with a poor prognosis after chemotherapy, is considered to be a biomarker for breast cancer. 5-Fluorouracil $(5 \mathrm{FU})$ is a nucleoside analog and is used as an anti-tumor agent in patients whose plasma dCyd concentrations are increased. Because both $\mathrm{dCyd}$ and $5 \mathrm{FU}$ are pyrimidine analogues, it is possible that they have pharmacokinetic/ pharmacodynamic interaction, by which the anti-cancer efficacy of 5FU would be reduced. Here, we examined the effects of dCyd on the cytotoxicity of 5FU on mouse myeloma SP2/0-Ag14 (SP2/0) cells lacking hypoxanthine-guanine-phosphoribosyl transferase (HGPRT) and RH4 hybridomas with HGPRT under asynchronized conditions. The reduced cell viability by $5 \mathrm{FU}$ was restored by co-, but not pre-, treatment of dCyd in both SP2/0 and RH4 cells, but this effect in the former tended to be greater than that in the latter, suggesting a possible involvement of HGPRT in the interaction, although this might not be a major mechanism. Moreover, dCyd administration to SP2/0 myeloma-bearing mice tended to shorten their 5FU-induced prolonged survival in vivo. Collectively, these results indicate that dCyd decreases the anti-tumor efficacy of $5 \mathrm{FU}$ and that a metabolic pathway via HGPRT is involved partially in this interaction. The evaluation of $\mathrm{dCyd}$ as a biomarker is believed to provide valuable information for effective and safe chemotherapy with $5 \mathrm{FU}$.

Key words 2'-deoxycytidine; 5-fluorouracil; biomarker; hypoxanthine-guanine-phosphoribosyl transferase; cell viability; survival

Recently, various kinds of biomarkers have been found to be useful for the diagnosis and therapy of diseases. The NIH definition of a biomarker is "a characteristic that is objectively measured and evaluated as an indicator of normal biological processes, pathogenic processes or pharmacologic responses to a therapeutic intervention." ${ }^{1)}$ Specifically, tumor markers have been essential in the early diagnosis and follow-up of therapy on special malignancies. For example, carcinoembryonic antigen (CEA), prostate specific antigen (PSA) and CA 19-9 are tumor-associated antigens or tumorspecific antigens that are increased in the sera of patients with colorectal cancer, prostatic cancer or pancreatic carcinoma, respectively, ${ }^{2-4)}$ and Bence-Jones protein ${ }^{5)}$ and homovanillic and vanillylmandelic acid ${ }^{6}$ are specific products generated from myeloma and neuroblastoma, respectively.

Yoshioka et al. ${ }^{7)}$ found that 2 -deoxycytidine (dCyd) was increased in the plasma of Egyptian patients with breast cancer who were treated with cyclophosphamide-methotrexate (MTX)-5-fluorouracil (5FU) (CMF combination chemotherapy) after surgery and still had a poor prognosis, while it was not increased in those with a good prognosis. In view of these findings, we proposed that $\mathrm{dCyd}$ might be a biomarker for breast cancer. Moreover, Ahmed et al. ${ }^{8)}$ confirmed that dCyd levels were correlated well with the number of chemotherapeutic courses and were higher in patients with positive lymph node metastases. Elstein et al. ${ }^{9)}$ reported that dCyd decreased the incidence of 5FU-induced apoptosis and that dCyd significantly mitigated 5FU-induced cell growth inhibition in synchronized murine erythroleukemic cells. Because dCyd is a pyrimidine, we postulate that increased levels of dCyd in such patients might diminish the anti-tumor effects of anti-metabolic chemotherapeutic agents, especially nucleoside analogs. The CMF combination chemotherapy includes the pyrimidine analog $5 \mathrm{FU}$, by which both $\mathrm{DNA}^{10-13}$ and $\mathrm{RNA}^{14,15)}$ synthesis are inhibited (Chart 1) ${ }^{16,17)}$; thus, there is a possibility that the poor diagnosis in such patients might be due to an interaction between dCyd and 5FU that results in the decreased anti-tumor efficacy of 5FU.

Peterson et al. ${ }^{18)}$ reported that the treatment of S-phase arrested Chinese hamster V76 cells with 5-fluoro-2'-deoxyuridine (FdUrd), which is an anabolite of 5FU by thymidine phosphoryrase (TP), stimulated 8-azaguanine-resistance and increased the mutation frequencies by about two-fold greater than the spontaneous ones. In the presence of $\mathrm{dCyd}$, furthermore, such mutation frequencies were increased, and the FdUrd-sensitivity was reduced in 8-azaguanine-resistance cells, which lack the essential hypoxanthine-guanine-phosphoribosyl transferase (HGPRT) functions in the salvage synthesis of purine nucleotides. However, considering the clinical situations, it is important to know whether an interaction between dCyd and 5FU occurs in asynchronized cells. In the present study, we examined the effects of dCyd on the cytotoxicity of 5FU against the viability of asynchronized cells.

We used SP2/0-Ag14 (SP2/0) mouse myeloma cells lacking HGPRT (8-azaguanine-resistance) and RH4 ${ }^{19)}$ hybridomas, which have HGPRT due to the fusion of medial iliac lymph-node cells with SP2/0 cells. ${ }^{20)}$ In these cells, differences in the nucleoside synthesis pathway of MTX, an antimetabolite, are reported to show a significant anti-tumor activity against SP2/0, but not RH4, cells. ${ }^{21,22)}$ Thus, the use of these different types of cells should provide valuable information for understanding the interaction between $\mathrm{dCyd}$ and $5 \mathrm{FU}$.

Here, we report that co-, but not pre-, treatment of dCyd diminishes the cytotoxic effect of 5FU in both SP2/0 and RH4 cells under asynchronized conditions in vitro and that HGPRT might be involved in this interaction. In the in vivo study, co-administration of dCyd tended to shorten the prolonged survival of mice treated by 5FU. These results indicate that evaluation of dCyd in plasma should be a useful 




Chart 1. Pyrimidine Nucleotide Synthesis Pathway

dCyd, 2'-deoxycytidine; MTX, methotrexate; 5FU, 5-fluorouracil; FdUrd, 5-fluoro-2'-deoxyuridine; TP, thymidine phosphorylase; TS, thymidylate synthase; (F)dUMP, (fluoro)deoxyuridine monophosphate; dTMP, thymidine monophosphate; dTTP, thymidine triphosphate; FUTP, fluorouridine triphosphate; $\mathrm{CH}_{2}$-THF, 5,10 -methylene-tetrahydroforate; PRPP, phosphoribosyl pyrophosphate.

biomarker not only for diagnosis/prognosis but also for the prediction of $5 \mathrm{FU}$ sensitivity in breast cancer patients.

\section{MATERIALS AND METHODS}

Chemicals dCyd and 5FU were obtained from Sigma (St. Louis, MO, U.S.A.). RPMI 1640 medium (RPMI) was obtained from Nikken Biomedical Laboratory (Kyoto). Fetal bovine serum (FBS) was from Sanko Junyaku (Tokyo). Plastic tissue culture dishes of $100-\mathrm{mm}$ diameter were from IWAKI Co. (Tokyo). Penicillin and streptomycin were obtained from Meiji Seika Co. (Tokyo). Phosphate buffered saline (PBS) was from Takara Shuzo Co., Ltd. (Shiga). Trypan blue was from Wako Pure Chemical Ind., Ltd. (Osaka). A $0.22-\mu \mathrm{m}$ Filter (Millex ${ }^{\mathrm{TM}}-\mathrm{GV}$ ) was purchased from Millipore Corporation (Bedford, MA, U.S.A.).

Cell Cultures of SP2/0-Ag14 Myeloma Cells and RH4 Hybridomas SP2/0-Ag14 mouse myeloma cells (SP2/0) were obtained from the Collection of Cancer Cell Lines (National Institute of Hygenic Science, Tokyo). RH4 hybridoma was produced in our laboratory as reported previously. ${ }^{19)}$ These cells were maintained in culture in RPMI containing $10 \%$ heat-inactivated FBS, penicillin at $100 \mathrm{U} / \mathrm{ml}$ and streptomycin at $100 \mu \mathrm{U} / \mathrm{ml}$. The cells were grown in $100-\mathrm{mm}$ plastic tissue culture dishes under a humidified atmosphere of $5 \% \mathrm{CO}_{2}$ at $37^{\circ} \mathrm{C}$.

Measurement of Cell Viability in Vitro dCyd and 5FU were dissolved in sterilized PBS at a given concentration and filtrated through a $0.22-\mu \mathrm{m}$ filter. Cell suspensions at a density of $1 \times 10^{5}$ cells $/ \mathrm{ml}$ were plated in $24-$ well $(1.0 \mathrm{ml}$ per well) plates containing a given dCyd- or $5 \mathrm{FU}$-containing test solution. After $72 \mathrm{~h}$ incubation, the cells were removed from each well by pipetting, and then their cell viability was evaluated by a trypan blue exclusion test. ${ }^{23)}$

As for pre-treatment with $\mathrm{dCyd}$, a SP $2 / 0$ cell suspension at a density of $1 \times 10^{4}$ cells/well $(200 \mu \mathrm{l} /$ well $)$ was plated in a 96-well plastic tissue culture plate and incubated at $37{ }^{\circ} \mathrm{C}$ for $24 \mathrm{~h}$. The medium was replaced with a fresh medium containing dCyd (pre-treatment) or PBS (co-treatment) at a given concentration. Four days later, the medium was replaced with a designated medium containing 5FU (pre-treatment) or dCyd $+5 \mathrm{FU}$ (co-treatment), and the cells were cultured for two days. Thereafter, the cell viability was evaluated (Fig. 3).

Survival of Mice Bearing SP2/0 Myeloma Tumors $\mathrm{BALB} / \mathrm{c}$ mice (male, five weeks old, weighing $20-25 \mathrm{~g}$ ) were obtained from Japan SLC (Hamamatsu). The drug administration schedule is shown in Fig. 4. SP2/0 myeloma cell suspensions were inoculated into the mice (intraperitoneally (i.p.), $1 \times 10^{6}$ cells/mouse). From the next day through the 8 th day, the test solution (control: PBS, dCyd: $0.1 \mathrm{mmol} / \mathrm{kg}$, 5FU: $0.15 \mathrm{mmol} / \mathrm{kg}, \mathrm{dCyd}+5 \mathrm{FU}$ : dCyd at $0.1 \mathrm{mmol} / \mathrm{kg}+$ $5 \mathrm{FU}$ at $0.15 \mathrm{mmol} / \mathrm{kg}$ ) was administered intraperitoneally on a daily basis, and the survival was evaluated.

The protocol used here was performed according to the guidelines of the Japanese Society for Pharmacology and was approved by the Committee for Ethical Use of Experimental Animals at Setsunan University.

Statistical Analysis of Cell Growth Data were analyzed by the $F$-test for variance and the Student's $t$-test using the DA-stat program for significance. Survival curves were calculated using the Kaplan-Meier method, and differences in survival periods were analyzed with the log-rank test.

\section{RESULTS}

Effect of dCyd Co-treatment with 5FU on SP2/0 Cell Viability The effects of 5FU, both alone and in combination with $\mathrm{dCyd}(\mathrm{dCyd}+5 \mathrm{FU})$, on the viability of SP2/0 cells are shown in Fig. 1. 5FU dose-dependently decreased the cell viability. $1 \mathrm{~mm} \mathrm{dCyd}$ had no effect on the cell viability, and its combination with 5FU significantly increased the effects 

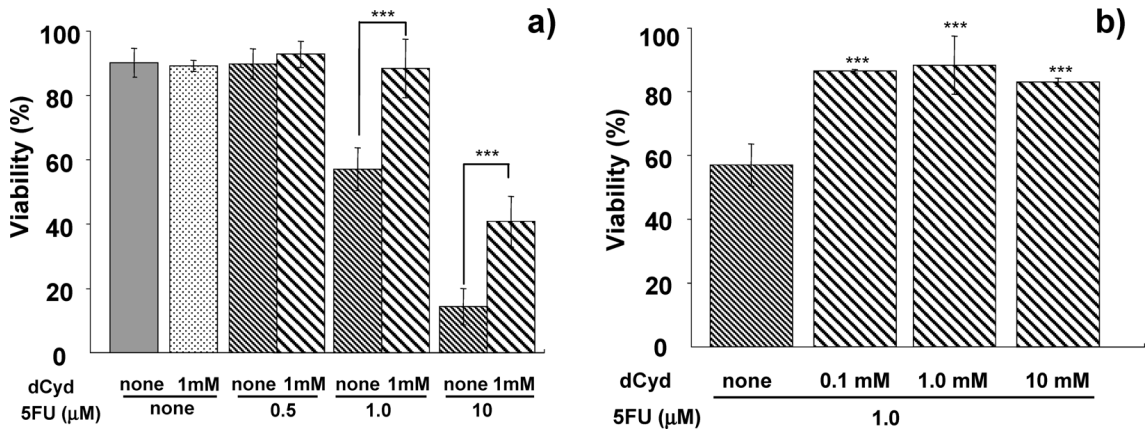

Fig. 1. Effect of Co-treatment of dCyd with 5FU on SP2/0 Cell Viability

(a) Cells were treated with the indicated concentrations of $5 \mathrm{FU}$ in the presence or absence of $1 \mathrm{~mm} \mathrm{dCyd} \mathrm{for} 72 \mathrm{~h}$. (b) Cells were treated with $1 \mu \mathrm{M} 5 \mathrm{FU}$ in the presence or absence of the indicated concentrations of dCyd for $72 \mathrm{~h}$. Thereafter, their cells viability was evaluated by a trypan blue exclusion test. Each value is represented as the mean \pm S.D. A significant difference from the level with $5 \mathrm{FU}$ alone is indicated by $* * * p<0.001$
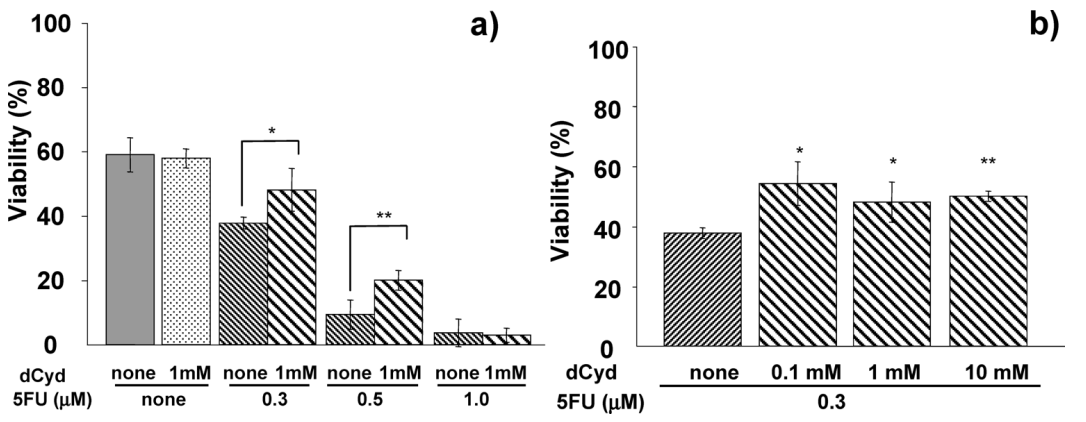

Fig. 2. Effect of Co-treatment of dCyd with 5FU on RH4 hybridoma Cell Viability

(a) Cells were treated with the indicated concentrations of 5FU in the presence or absence of $1 \mathrm{~mm}$ dCyd for $72 \mathrm{~h}$. (b) Cells were treated with $1 \mu \mathrm{M} 5 \mathrm{FU}$ in the presence or absence of the indicated concentrations of $\mathrm{dCyd}$ for $72 \mathrm{~h}$. Thereafter, their cells viability was evaluated by a trypan blue exclusion test. Each value is represented as the mean \pm S.D. Significant differences from the level with $5 \mathrm{FU}$ alone are indicated by $* p<0.05$, and $* * p<0.01$.

compared with the case of 5FU alone (Fig. 1a). At $1 \mu \mathrm{M}$ of $5 \mathrm{FU}$, dCyd in the concentration range from 0.1 to $10 \mathrm{~mm}$ increased the 5FU-induced decrease of cell viability by up to approximately $30 \%$, and the increment of cell viability with dCyd did not show dose-dependency (Fig. 1b).

Effect of dCyd Co-treatment with 5FU on RH4 Cell Viability As shown in Fig. 2a, there was a dose-dependent decrease of the viability of RH4 hybridoma cells by the $5 \mathrm{FU}$ treatment, and this cytotoxic effect in RH4 cells was observed at lower concentrations of $5 \mathrm{FU}$ than that in $\mathrm{SP} 2 / 0$ cells. As with the case of SP2/0 cells, dCyd showed the doseindependent increase on the $5 \mathrm{FU}(0.3 \mu \mathrm{M})$-treated cell viability at $0.1,1$ and $10 \mathrm{~mm}$ in RH4 cells, with increased effects on the viability of approximately $20 \%$ (Fig. 2 b).

Effect of dCyd Pre-treatment with 5FU on SP2/0 Cell Viability Co-treatment of dCyd with 5FU significantly increased the cell viability of SP $2 / 0$ cells compared to the case of 5FU alone. In contrast, the pre-treatment with dCyd for four days did not show any effect on the 5FU-induced decrease of SP2/0 cell viability (Fig. 3).

Effect of dCyd Treatment with 5FU on Survival of SP2/0-Bearing Mice With the transplantation of SP2/0 cells to mice, their survival was time-dependently decreased in the control and dCyd groups, with the mean survival times calculated as 25 and $24 \mathrm{~d}$, respectively. This survival time of mice was significantly increased to $48 \mathrm{~d}$ by the $5 \mathrm{FU}$ administration, and this prolonged survival tended to be decreased to $40 \mathrm{~d}$ by the co-administration of dCyd (Fig. 4).

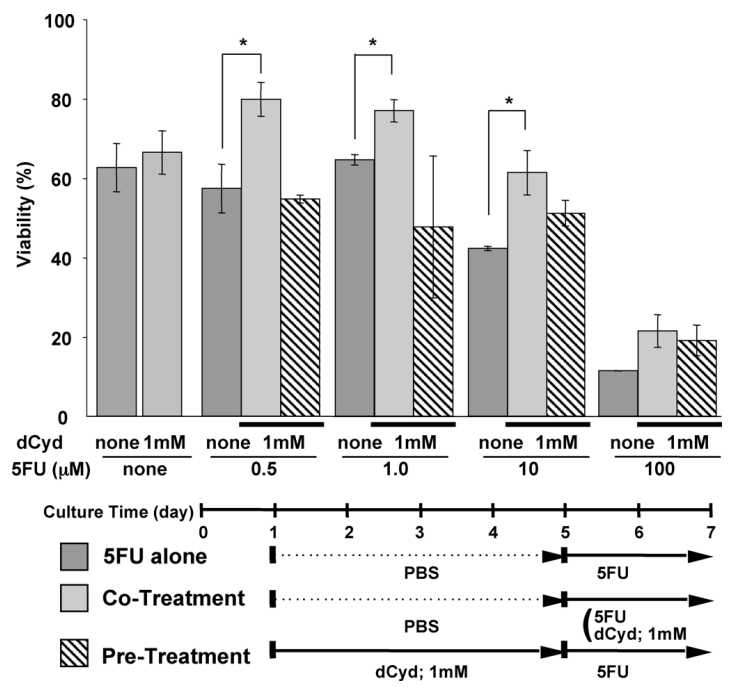

Fig. 3. Effect of Pre-treatment of dCyd with 5FU on SP2/0 Cell Viability After cells were pre-treated with $1 \mathrm{~mm}$ dCyd for four days, they were treated with the indicated concentrations of 5FU in the presence or absence of $1 \mathrm{~mm} \mathrm{dCyd} \mathrm{for} 72 \mathrm{~h}$ Thereafter, their cells viability was evaluated by a trypan blue exclusion test. Each value is represented as the mean \pm S.D. A significant difference from the level of $5 \mathrm{FU}$ alone is indicated by $* p<0.05$.

\section{DISCUSSION}

In this study, we examined whether dCyd had any effect on 5FU's anti-tumor activity in vitro and in vivo. Treatment with dCyd diminished the cytotoxic effect of 5FU in SP2/0 and RH4 cells in vitro, and the prolonged survival of SP2/0-bear- 


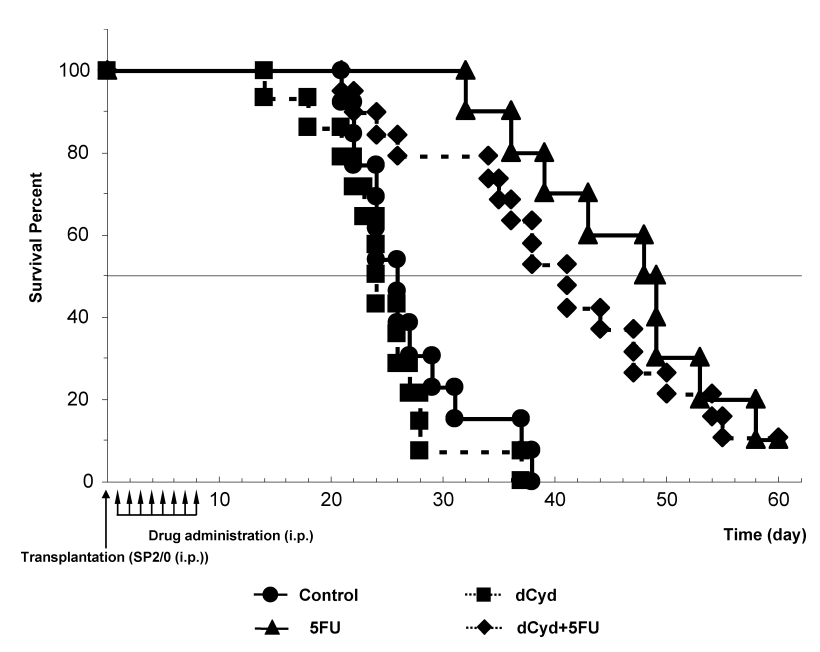

Fig. 4. Effect of Treatment of $5 F U$ and dCyd on the Survival of SP2/0 Cell-Bearing Mice

One day after the transplantation of SP2/0 cells to mice $\left(1 \times 10^{6}\right.$ cells/mouse, i.p. $)$, dCyd $(0.1 \mathrm{mmol} / \mathrm{kg}), 5 \mathrm{FU}(0.15 \mathrm{mmol} / \mathrm{kg})$ or dCyd $(0.1 \mathrm{mmol} / \mathrm{kg})$ plus $5 \mathrm{FU}(0.15$ $\mathrm{mmol} / \mathrm{kg}$ ) was administered (i.p.) to them for $8 \mathrm{~d}$. After the transplantation, the survival of the mice was observed daily, and survival curves were calculated by the KaplanMeier method.

ing mice receiving $5 \mathrm{FU}$ treatment was shortened by the coadministration of dCyd in vivo. These findings were obtained with asynchronized cells, differing from the study performed by Peterson et al. ${ }^{18)}$ with synchronized cells. Thus, it is suggested that in breast cancer patients with high plasma levels of dCyd, 5FU might not exhibit the anti-tumor effects as expected, resulting in poorer prognoses.

The following results obtained here might be important for clarifying the mechanism underlying the interaction between dCyd and 5FU: (1) RH4 cells had a higher sensitivity to 5FU than SP2/0 cells did, and (2) the recovery rate of cell viability with dCyd was greater in SP2/0 cells than in RH4 cells (Figs. $1,2)$. Since SP2/0 cells lack the HGPRT found in RH4 cells, we postulate that HGPRT might play an important role in the expression of the anti-tumor activity of 5FU, while it might have a minor role in the decrease of the cytotoxic effect of $5 \mathrm{FU}$ by dCyd. However, it was concluded that the contribution, if any at all, of HGPRT in this interaction was negligible. This conflicts with the report of Peterson et al., ${ }^{18)}$ in which the mutation frequency induced by the 5FU anabolite FdUrd is increased in synchronized HGPRT-lacking cells. Although the details are not clear, differences in the experimental conditions such as the drug treatment schedules, cell types, end points of evaluation, etc., are considered to be the causes of this discrepancy.

The decreasing effect of dCyd on 5FU anti-tumor activity was observed under the co-treatment condition, suggesting the possibility of their interaction during the cellular uptake of dCyd (Chart $1 \mathrm{I}$ ) and 5FU (Chart 1 II). It is reported that $5 \mathrm{FU}$ is transported by organic anion transporter 2 (OAT2), ${ }^{24)}$ $\mathrm{OAT}^{25)}$ and sodium-dependent nucleobase transporter $\left.1,{ }^{26}\right)$ and no contribution of nucleoside transporters (by which nucleobases are taken up into cells) has been demonstrated for its transport. ${ }^{27,28)}$ On the other hand, dCyd is a pyrimidine nucleoside analogue, so the nucleoside transport system may mediate its cellular uptake, but there is no available information on its membrane transport. Thus, clarification of the transport system for dCyd should lead us to a better under- standing of the interaction between dCyd and 5FU, and this project is now underway in our laboratory.

In conclusion, we showed that dCyd decreased the antitumor effects of $5 \mathrm{FU}$ in in vitro and in vivo experiments. These findings suggest that the evaluation of dCyd in plasma should be a useful biomarker not only for diagnosis/prognosis but also for the prediction of 5FU sensitivity in breast cancer patients.

Acknowledgments The authors thank Ms. Kobayashi M., Ms. Tokunoh C., Ms. Tominaga A. and Ms. Yabushita A. for technical assistance. We appreciate professor EL-Merzabani M. of the National Cancer Institute, Cairo University, for his critical advice.

\section{REFERENCES}

1) Biomarkers Definition Working Group, Clin. Pharmacol. Ther, 69, $89-95$ (2001).

2) Bhatnagar J., Tewari H. B., Bhatnagar M., Austin G. E., Anticancer Res., 19, 2181-2187 (1999).

3) Balk S. P., Ko Y. J., Bubley G. J., J. Clin. Oncol., 21, 383-391 (2003).

4) Dianxu F., Shengdao Z., Tianquan H., Yu J., Ruoqing L., Zurong Y., Xuezhi W., Pancreas, 25, 336-341 (2002).

5) Bradwell A. R., Carr-Smith H. D., Mead G. P., Harvey T. C., Drayson M. T., Lancet, 361, 489-491 (2003).

6) Kuroda M., Sugi M., Ikeda T., Fujimoto M., Yokomori K., Amano J., Yoshioka M., Biog. Amines, 7, 257-263 (1990).

7) Yoshioka M., Avu-Zeid M., Kubo T., EL-Merzabani M., Biol. Pharm. Bull., 17, 169-174 (1994).

8) Ahmed W. A., Abu-Zeid M., Yoshioka M., Khaled H., EL-Merzabani M., J. Egypt Ger. Soc. Zool., 35, 249-263 (2001).

9) Elstein K. H., Mole M. L., Setzer R. W., Zucker R. M., Kavlock R. J., Rogers J. M., Lau C., Toxicol. Applied Pharmacol., 146, 29-39 (1997).

10) Valeriote F., Santinelli G.., Pharmacol. Ther, 24, 107-132 (1984).

11) Schuetz J. D., Wallace H. J., Diasio R. B., Cancer Res., 44, 13581363 (1984).

12) O'Dwyer P. J., King S. A., Hoth D. F., Leyland-Jones B., Cancer Res., 47, 3911-3919 (1987).

13) Kyprianou N., Isaacs J. T., Biochem. Biophys. Res. Commun., 165, 73 -81 (1989).

14) Parker W. B., Cheng Y. C., Pharmacol. Ther., 48, 381-395 (1990).

15) Pinendo H. M., Peters G. F. J., J. Clin. Oncol., 6, 1653-1664 (1988).

16) Peters G. J., Backus H. H. J., Freemantle S., van Triest B., CodacciPisanelli G., van der Wilt C. L., Smid K., Lunec J., Calvert A. H., Marsh S., McLeod H. L., Bloemena E., Meijer S., Jansen G., van Groeningen C. J., Pinedo H. M., Biochim. Biophys. Acta, 1587, 194205 (2002).

17) Grant S., Cadman E., Cancer Res., 42, 3550-3556 (1982).

18) Peterson A. R., Peterson H., Danenberg P. V., Biochem. Biophys. Res. Commun., 110, 573-577 (1983).

19) Darwish I., Akizawa T., Hirose K., Omura K., EL-Rabbat N., Yoshioka M., Analytica Chimica Acta, 365, 121-128 (1998).

20) Shulman M., Wilde C. D., Kohler G., Nature (London), 276, 269-270 (1978).

21) Littlefield J. W., Biochim. Biophys. Acta, 95, 14-22 (1965).

22) Littlefield J. W., Science, 145, $709-710$ (1964).

23) Rossi L., Serafini S., Schiavano G. F., Casabianca A., Vallanti G., Chiarantini L., Magnani M., Biochem. J., 344, 915-920 (1999).

24) Kobayashi Y., Ohshiro N., Sakai R., Ohbayashi M., Kohyama N., Yamamoto T., J. Pharm. Pharmacol., 57, 573-578 (2005).

25) Kobayashi Y., Ohshiro N., Tsuchiya A., Kohyama N., Ohbayashi M., Yamamoto T., Drug Metab. Dispos., 32, 479_483 (2004).

26) Yamamoto S., Inoue K., Murata T., Kamigaso S., Yasujima T., Maeda J. Y., Yoshida Y., Ohta K. Y., Yuasa H., J. Biol. Chem., 285, 65226531 (2010).

27) Yao S. Y. M., Ng A. M. L., Vickers M. F., Sundaram M., Cass C. E., Baldwin S. A., Young J. D., J. Biol. Chem., 277, 24938-24948 (2002).

28) Tsujie M., Nakamori S., Nakahira S., Takahashi Y., Hayashi N., Okami J., Nagano H., Dono K., Umeshita K., Sakon M., Monden M., Anticancer Res., 27, 2241-2249 (2007). 